\title{
A NOTE ON FIXED-POINT-FREE SOLVABLE OPERATOR GROUPS
}

\section{FLETCHER GROSS ${ }^{1}$}

Let $A$ be a finite group and let $n$ be the number of primes, including multiplicities, which divide the order of $A$. Suppose further that $G$ is a finite solvable group which admits $A$ as a fixed-point-free operator group and such that $(|A|,|G|)=1$. Under these assumptions it has been conjectured that $l(G)$, the nilpotent length of $G$, is at most $n$, while $l_{p}(G)$, the $p$-length of $G$, is at most $[(n+1) / 2]$. These inequalities have been verified and shown to be best-possible in certain special cases ([3]-[5], [1]). It is the purpose of this note to show that these conjectured upper bounds are actually obtained provided $A$ is solvable. Specifically we will prove

THEOREM 1. Let $A$ be a finite solvable group with $n$ the number of primes, including multiplicities, which divide $|A|$. Let $p$ be a prime not dividing $|A|$. Then there exists a finite solvable group $G$ of order prime to $|A|$ which admits $A$ as a fixed-point-free operator group and such that

$$
l(G)=n, \quad l_{p}(G)=[(n+1) / 2] .
$$

We now adopt the convention that all groups referred to are finite. $\pi^{\prime}(A)$ denotes the set of primes which do not divide $|A|$ while $\psi(A)$ denotes the number of primes, including multiplicities, which divide $|A|$. Other than the following definition, the rest of the notation is standard.

Definition. If $A$ is a group and $p, q$ are distinct primes in $\pi^{\prime}(A)$, then $A$ is said to satisfy $E(p, q, n)$ if there exist two $p, q$-groups $G$ and $H$ such that

(i) $A$ operates in a fixed-point-free manner on both $G$ and $H$.

(ii) $l(G)=l(H)=n$.

(iii) $O_{p}(G)=O_{q}(H)=1$.

REMARK. (ii) and (iii) imply that

$$
l_{q}(G)=l_{p}(H)=[(n+1) / 2] .
$$

Lemma. If $A$ is cyclic of prime order and $p, q$ are distinct members of $\pi^{\prime}(A)$, then $A$ satisfies $E(p, q, 1)$.

Received by the editors July 22, 1967.

${ }^{1}$ Research supported in part by a grant from the National Research Council of Canada. 
Proof. Let $K$ be a finite field of characteristic $q$ containing a primitive $|A|$ th root of unity $\theta$. Let $G$ be the additive group of $K$ and let $A$ operate on $G$ by multiplication by $\theta$. $H$ is constructed in a similar manner.

Theorem 2. If $B<A$ and $B$ satisfies $E(p, q, n)$ for $p, q \in \pi^{\prime}(A)$, then $A$ satisfies $E(p, q, n)$.

Proof. Let $G$ be a $p, q$-group on which $B$ operates in a fixed-pointfree manner and such that $l(G)=n$ and $O_{p}(G)=1$. Let $x_{1}, x_{2}, \cdots, x_{m}$ be a complete set of representatives for the left cosets of $B$ in $A$. Next for $i=1,2, \cdots, m$ let $G_{i}$ be the set of all ordered pairs $(g, i)$ where $g \in G$. Under the multiplication $\left(g_{1}, i\right)\left(g_{2}, i\right)=\left(g_{1} g_{2}, i\right), G_{i}$ becomes a group isomorphic to $G$. Finally let $G^{*}=G_{1} \times G_{2} \times \cdots \times G_{m}$. If $x \in A$, define $(g, i)^{x}$ to be $\left(g^{y}, j\right)$ where $y \in B$ and $x_{i} x=y x_{j}$. In this way $G^{*}$ admits $A$ as a fixed-point-free operator group. Obviously $l\left(G^{*}\right)=n$ and $O_{p}\left(G^{*}\right)=1$.

Theorem 3. Let $B \triangle A$ and $B$ satisfy $E(p, q, m)$ while $A$ satisfies $E(p, q, n)$. Then $A$ satisfies $E(p, q, m+n)$.

Proof. Let $G$ be a $p, q$-group which admits $B$ as a fixed-point-free operator group and such that $l(G)=m$ and $O_{p}(G)=1$. Use the method in the proof of the last theorem to construct a group $G^{*}$ isomorphic to the direct product of $[A: B]$ copies of $G$ such that $A$ operates in a fixed-point-free manner on $G^{*}$. From the fact that $B$ is normal in $A$, we easily conclude that $B$ is fixed-point-free on $G^{*}$.

Now $G^{*} / F_{m-1}\left(G^{*}\right)$ is either a $p$ - or a $q$-group. Thus there exists a $p, q$-group $H$ such that $A / B$ operates in a fixed-point-free manner on $H, l(H)=n$, and

$$
\left(\left|G^{*} / F_{m-1}\left(G^{*}\right)\right|,\left|F_{1}(H)\right|\right)=1 .
$$

We will consider $A$ as operating on $H$ by letting $B$ operate trivially.

Consider the group $G^{*} \sim H$, the wreath product of $G^{*}$ by $H$. $l\left(G^{*} \sim H\right)=m+n$ and $O_{p}\left(G^{*} \sim H\right)=1$ [2]. One way of representing $G^{*} \sim H$ is as follows: If $h \in H$, let $G_{h}^{*}=\left\{(g, h) \mid g \in G^{*}\right\}$, and define a multiplication in $G_{h}^{*}$ by $\left(g_{1}, h\right)\left(g_{2}, h\right)=\left(g_{1} g_{2}, h\right)$. Thus $G_{h}^{*}$ is a group isomorphic to $G^{*}$. Let $\bar{G}$ be the direct product of the $G_{h}^{*}$ for all $h \in H$. $H$ operates on $\bar{G}$ according to the rule $\left(g, h_{1}\right)^{h}=\left(g, h_{1} h\right)$. Then $G^{*} \sim H$ is the semidirect product $\bar{G} H$. $A$ operates on $\bar{G}$ as follows:

$$
(g, h)^{x}=\left(g^{x}, h^{x}\right) \quad \text { if } x \in A .
$$

It follows that $\left(g, h_{1}\right)^{x^{-1} h x}=\left(g, h_{1}\right)^{\left(h^{x}\right)}$ for $x \in A, h \in H$. Thus we may consider $A$ as operating on $\bar{G} H$. 
Clearly $A$ is fixed-point-free on $\bar{G} H / \bar{G} \cong H$. If $y \in B$, then $(g, h)^{y}$ $=\left(g^{y}, h\right)$. Since $B$ is fixed-point-free on $G^{*}$, we easily conclude that $B$ is fixed-point-free on $\bar{G}$. Thus $A$ is fixed-point-free on both $\bar{G}$ and $\bar{G} H / \bar{G}$. This finishes the proof.

Corollary. If $A$ is solvable and $p, q$ are distinct members of $\pi^{\prime}(A)$, then $A$ satisfies $E(p, q, \psi(A))$.

Proof. We use induction on $\psi(A)$. If $B$ is a maximal normal subgroup of $A$, then $|A / B|$ is a prime and $\psi(B)=\psi(A)-1$. By induction $B$ satisfies $E(p, q, \psi(A)-1)$ while $A / B$ satisfies $E(p, q, 1)$. The theorem now implies that $A$ satisfies $E(p, q, \psi(A))$. Clearly Theorem 1 is an immediate consequence of this corollary.

\section{REFERENCES}

1. F. Gross, Solvable groups admitting a fixed-point-free automorphism of prime power order, Proc. Amer. Math. Soc. 17 (1966), 1440-1446.

2. P. Hall and G. Higman, On the p-length of p-soluble groups and reduction theorems for Burnside's problem, Proc. London Math. Soc. (3) 6 (1956), 1-42.

3. F. Hoffman, Nilpotent height of finite groups admitting fixed-point-free automorphisms, Math. Z. 85 (1964), 260-267.

4. E. Shult, On groups admitting fixed-point-free abelian groups, Illinois J. Math. 9 (1965), 701-720.

5. - Nilpotence of the commutator subgroup in groups admitting fixed point free operator groups, Pacific J. Math. 17 (1966), 323-347.

UNIVERSITY OF UTAH 